\title{
Empirical Evaluation of 20 Web Form Optimization Guidelines
}

Mirjam Seckler

University of Basel, Switzerland University of Basel, Switzerland

Dept. of Psychology

Cognitive Psych. \& Methodology Cognitive Psych. \& Methodology

Missionsstr. 62a, 4055 Basel Missionsstr. 62a, 4055 Basel

mirjam.seckler@unibas.ch

klaus.opwis@unibas.ch

Silvia Heinz

Alexandre N. Tuch

University of Basel, Switzerland University of Copenhagen, Denmark Dept. of Psychology Dept. of Computer Science

Cognitive Psych. \& Methodology Njalsgade 128, 2300 Copenhagen

Missionsstr. 62a, 4055 Basel a.tuch@unibas.ch

silvia.heinz@unibas.ch

\section{J avier A. Bargas-Avila}

Google/YouTube User Experience

Research

Brandschenkestr. 110, 8002

Zurich, Switzerland

bargas@google.com

\author{
Abstract \\ Most websites use interactive online forms as a main \\ contact point to users. Recently, many publications aim \\ at optimizing web forms. In contrast to former research \\ that focused at the evaluation of single guidelines, the \\ present study shows in a controlled lab experiment with \\ $\mathrm{n}=23$ participants the combined effectiveness of 20 \\ guidelines on real company web forms. Results indicate \\ that optimized web forms lead to faster completion \\ times, less form submission trials, fewer eye fixations \\ and higher user satisfaction in comparison to the \\ original forms.
}

\section{Author Keywords}

Web Forms; Form Guidelines; Form Evaluation

\section{ACM Classification Keywords}

H.3.4 Systems and Software: Performance evaluation;

H.5.2 User Interfaces: Evaluation/methodology

H.5.2 User Interfaces: Interaction styles

\section{I ntroduction}

Since its early beginning, the Internets technological development has come a long way. Hypertext, the core component of the World Wide Web that helped breaking the linearity of text, was quickly expanded by many powerful technologies that added high levels of interactivity and different types of media. 
Despite this evolution, web forms remain one of the core interaction elements between users and website owners

[15]. Web forms are used as registration forms to

subscribe to services and communities, checkout forms to initiate transactions between users and companies, or data input forms to search or share information [16]. In this sense, they can be regarded as gatekeepers between website owners and users. As a consequence of this gatekeeper role, any kind of problems and obstacles users may experience while filling in forms, may lead to

increased drop-out rates and data loss for the provider of the forms. Therefore website developers must pay special attention to optimize their forms and make them as usable as possible.

In the last years, an increasing number of publications looked at a broad range of aspects surrounding web form interaction, to help developers optimize their forms. These include topics such as error message optimization [15], error prevention $[6,14]$, optimization of form interaction elements $[4,5,7,8]$, optimization for different devices [11], or accessibility optimization [13].

These studies share light on selected aspects of web form interaction, and in the last years there have been several approaches to gather the various sources of knowledge in this field and compile them as checklists [10] or guidelines [3]. The latter presents 20 rules that aim at optimizing form content, layout, input types, error handling and submission

Currently there is no empirical study that applies these guidelines in a holistic approach to web forms and shows if there are effects on efficiency, effectiveness and user satisfaction.
It is this gap that we aim to close with our ongoing study. The main research goal is to conduct an empirica

experiment to understand if optimizing web forms using current guidelines lead to a significant improvement of total user experience. For this we selected a sample of existing web forms from popular news web sites, and optimized them according to the 20 guidelines presented in [3]. In a controlled lab experiment we let participants use the original and optimized forms, while measuring efficiency, effectiveness and user satisfaction. We

expected all optimized forms to perform better than their original counterpart.

\section{Method}

Study design

In order to investigate as to how the implementation of the form guidelines of [3] improve user experience, we conducted an eye tracking lab study, where participants had to fill in either the original or an optimized version of an online form (within-subject design). User experience was measured by means of objective data such as task completion time, effectiveness of corrections and number of fixations, but also by subjective ratings on satisfaction, usability and mental load.

\section{Participants}

In total 23 participants ( 12 female) took part in the study. Eleven were assigned to the original form and 12 to the optimized form condition. The mean age of the participants was 30 years $(S D=12)$ and all were experienced Internet users $(M=5.4, S D=0.85$ with 1 = "no experience"; 7 = "expert")

Selection and optimization of web forms By screening www.ranking.com for high traffic websites we ensured to get realistic and commonly 
used web forms. Thereby we focused on the top ranked German-speaking newspapers and magazines, which provide an online registration form $(\mathrm{N}=23)$. Subsequently, we evaluated all forms in regard to the 20 form design guidelines provided by [3]. Two raters independently coded for each form whether a guideline was violated or not (Cohen's

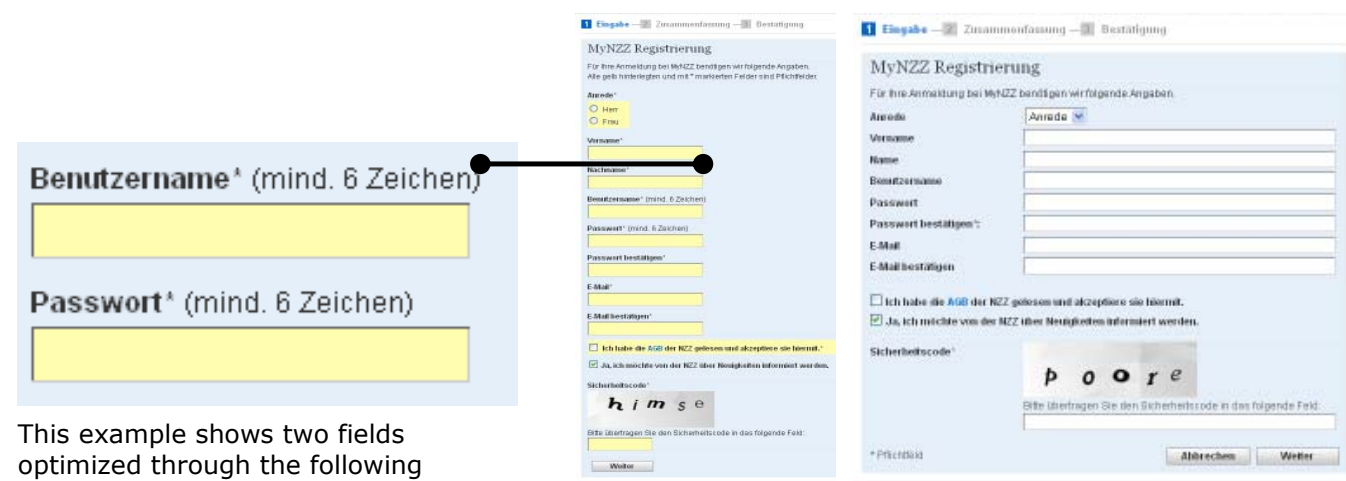

optimized through the following

three guidelines [3]:

- Guideline 4: If possible and reasonable, separate required from reasonable, separate required from optional fields and use color and

- Guideline 5: To enable people to fill in a form as fast as possible, fill in a form as fast as possible, place the labels above the

corresponding input fields.

required in a specific format, state this in advance communicating the (format specification) without an additional example.
Figure 1. Optimized form on the left, original form on the right side.

kappa $=0.70)$. Additionally, 14 usability experts rated each of the 20 guidelines on how serious the consequences of a violation would be for a potential user (from $1=$ not serious to $5=$ serious;

Cronbach's $\alpha=.90$ ).

Based on the two ratings we ranked the forms from good to bad and selected three for our main study: One of rather good quality (Spiegel.de; ranked \#11), one of medium quality (nzz.ch; \#13) and one of rather bad quality (sueddeutsche.de; \#18). We did not select any form from the first third (rank 1 to 8 ), since these forms had only minor violations and hence little potential for improvement. By means of reverse engineering we built a copy of the original form and an optimized version according to the 20 guidelines (see Fig. 1 for an example). Thereby, the number of optional and required fields was retained.

Measurements

User experience was assessed by means of user performance and subjective ratings. User performance: time efficiency (task completion time, number of fixations) and effectiveness of corrections (number of trials to submit a form). Eye-tracking data were collected with a SMI RED eye-tracker using Experiment Center 3.2.17 software, sampling rate $=60$ $\mathrm{Hz}$. Subjective Ratings: general satisfaction, NASA Task Load Index (TLX) [9], SUS [2], After Scenario Questionnaire (ASQ) [12], Form Usability Scale (FUS) [1] and interview data.

\section{Procedure}

After filling in a baseline form, participants were randomly assigned to one of the experimental conditions (original vs. optimized). The baseline form was the same for all participants and served as practice trial. Participants then were forwarded to a landing page that featured general information about one of the selected newspapers and a link to the registration form. Participants were instructed to follow that link and to register for the online magazine. After filling in and successfully submitting the form, they had to evaluate the form by means of a set of questionnaires. This procedure was repeated for all online forms (in a random sequence). In the end participants were interviewed on how they experienced the interaction with the forms. In these interviews we focused on aspects of the form that participants found especially annoying or easy to fill in. 


\begin{tabular}{|l|c|c|c|}
\hline Form & Trials & Orig. & Opt. \\
\hline Suedd. & 1 & 3 & 10 \\
\hline & $\geq 2$ & 8 & 2 \\
\hline NZZ & 1 & 4 & 9 \\
\hline & $\geq 2$ & 7 & 3 \\
\hline Spiegel & 1 & 7 & 10 \\
\hline & $\geq 2$ & 4 & 2 \\
\hline
\end{tabular}

Table 1. Number of trials until form was successfully submitted.

\begin{tabular}{|l|c|c|}
\hline & $\begin{array}{c}\text { Original } \\
\mathbf{n = 1 1} \\
\mathbf{M} \text { (SD) }\end{array}$ & $\begin{array}{c}\text { Optimized } \\
\mathbf{n = 1 2} \\
\mathbf{M} \text { (SD) }\end{array}$ \\
\hline Suedd. & $113(40)$ & $90(26)$ \\
\hline NZZ & $99(60)$ & $71(20)$ \\
\hline Spiegel & $103(89)$ & $91(32)$ \\
\hline
\end{tabular}

Table 2. Average task completion time in seconds.

\begin{tabular}{|l|c|c|}
\hline & $\begin{array}{c}\text { Original } \\
\mathbf{n = 1 0} \\
\mathbf{M} \text { (SD) }\end{array}$ & $\begin{array}{c}\text { Optimized } \\
\mathbf{n = 1 0 - 1 2} \\
\mathbf{M} \text { (SD) }\end{array}$ \\
\hline Suedd. & $182(58)$ & $121(28)$ \\
\hline NZZ & $121(46)$ & $88(20)$ \\
\hline Spiegel & $118(38)$ & $118(48)$ \\
\hline
\end{tabular}

Table 3. Number of fixations until the form was successfully filled in.

\section{Results}

User Performance

As expected, users predominantly performed better with the optimized version of the forms. In two out of three forms they needed fewer trials to successfully submitting the form (until all fields were filled in correctly): Suddeutsche $\left(x^{2}=7.34, p=.003\right), N Z Z\left(x^{2}\right.$ $=3.49, p=.031)$, and Spiegel $\left(x^{2}=1.16, p=.142\right)$. See Table 1 for corresponding figures.

Also in regard to task completion time the optimized version of the forms performed better than the original ones. An independent sample t-test hints at potential effects with large magnitudes for the Sueddeutsche $(\mathrm{t}(21)=1.64, \mathrm{p}=.058$, Cohen's $\mathrm{d}=.72)$ and the NZZ form $(t(21)=1.63, p=.059, d=.71)$. No effect was found for the Spiegel form $(t(21)=0.10, p=.462, d=$ $.04)$. Note that the $p$-values don't reach significance due to the small sample size. Table 2 shows the average task completion times for all forms.

Moreover, the eye tracking data show a similar picture (see Table 3). Participants assigned to the optimized form condition needed fewer fixations to successfully filling in the forms, with exception for the Spiegel form: Sueddeutsche $(t(20)=3.07, p=.005, d=1.37), N Z Z$ $(\mathrm{t}(18)=2.04, \mathrm{p}=.028, \mathrm{~d}=.91)$, and Spiegel $(\mathrm{t}(18)=$ $0.02, p=.492, d=.01$ ).

\section{Subjective Ratings}

In order to account for inter-individual differences we first baseline-corrected all questionnaire ratings by subtracting the ratings of the forms from the ratings of the baseline form (which was for all participants the same). These scores were then used to compare the optimized vs. the original versions of the forms by means of independent t-tests.
As expected all optimized forms received better ratings than their original counter parts (see Table 4).

Participants perceived the optimized versions as more usable (ASQ, FUS, SUS), as less demanding (NASATLX) and were more satisfied with them (Satisfaction). Although not all comparisons are significant, effect size calculations (Cohen's d) revealed that most effects were of medium to large magnitude $(d=.50$,

respectively $d=.80$ ). This means that increasing our sample size to 20-34 participants per group would make most of the results significant. Only the SUS showed small effects $(d=.20)$ for two forms. According to a power analysis one would require a sample of 71 to 148 participants per group to achieve a significant result for the SUS.

\begin{tabular}{|l|l|r|c|c|}
\hline Scale & Form & I mprov. & p & d \\
\hline ASQ & Suedd. & $16 \%$ & .05 & .74 \\
\hline & NZZ & $23 \%$ & .03 & .90 \\
\hline & Spiegel & $14 \%$ & .07 & .67 \\
\hline FUS & Suedd. & $9 \%$ & .09 & .61 \\
\hline & NZZ & $20 \%$ & .00 & 1.28 \\
\hline & Spiegel & $12 \%$ & .04 & .79 \\
\hline SUS & Suedd. & $5 \%$ & .26 & .29 \\
\hline & NZZ & $16 \%$ & .02 & .94 \\
\hline & Spiegel & $8 \%$ & .17 & .42 \\
\hline NASA-TLX & Suedd. & $-8 \%$ & .05 & .75 \\
\hline & NZZ & $-8 \%$ & .05 & .73 \\
\hline & Spiegel & $-7 \%$ & .05 & .76 \\
\hline Satisfaction & Suedd. & $12 \%$ & .09 & .60 \\
\hline & NZZ & $21 \%$ & .02 & .99 \\
\hline & Spiegel & $20 \%$ & .04 & .82 \\
\hline
\end{tabular}

Table 4. Effects on subjective ratings: relative impact from the original to the optimized version of the forms. 


Form Usability Scale FUS
The FUS is a validated
questionnaire to measure the
usability of online forms [1].
It consists of 9 items each to
be rated on a Likert-Scale
ranging from 1 (strongly
disagree) to 6 (strongly
agree). The total FUS score is
obtained by computing the
mean of all items.
Items: (1) I perceived the
length of the form as
appropriate. (2) I was able to
fill in the form quickly. (3) I
perceived the order of the
questions in the form as
logical. (4) Mandatory fields
were clearly visible in the
form. (5) I always knew
which information was
expected of me. (6) I knew at
every input which rules I had
to stick to (e.g. possible
answer length, password re-
quirements). (7) In case of a
problem I was instructed by
an error message how to
solve the problem. ( 8 ) The
purpose and use of the form
was clear. (9) In general I
am satisfied with the form.

Interview data

The analysis of the interview data showed that the most mentioned issues are the layout of the forms, the identification of required and optional fields and, if indicated, format specifications. The most reported favorable factors of the optimized forms were therefore the clearly structured and concise layout, the arrangement and marking of required and optiona fields in separate groups and the format specification especially for passwords and usernames.

\section{Discussion}

This study showed that with the application of the web form optimization guidelines all three web forms were improved regarding user performance and subjective ratings. Eye-tracking data revealed furthermore that the original forms needed more fixations than the optimized forms. Most of the effects were significant even with a small sample size and in addition effect sizes showed mostly medium to large magnitude.

Our findings highlight the importance for web designers to apply web form guidelines. A closer look at the form submission trials shows that there is great potential for increasing the number of successful first form submissions by applying form guidelines. Thereby website owners can minimize the risk that the user leaves their site as a consequence of an unsuccessful form submission. Furthermore, data for the task completion time shows an improvement by 10 to $25 \%$. Finally, subjective ratings could be improved by up to $23 \%$. To sum up, the effort to optimize the web forms is relatively low compared to the impact on user experience as shown by these results.

\section{Further work}

In the future we will continue this study adding more participants and extend the analysis of the data (e.g. explore the correlation between subjective and objective data). It would be interesting to know on more detailed level how the guidelines work. It also may be worth to explore the implications outside the lab and perform extended A/B testings in collaboration with website owners. Moreover, we could explore if the findings from this study can be replicated with other type of forms (e.g. longer forms with more than one site or other use cases such as web shops or social networks). Additionally, from an economical standpoint it would be important to know how the guidelines influence not only user experience aspects, but also conversion rates.

\section{Conclusion}

This study shows how form optimization guidelines can help improve the user experience of web forms. In contrast to former research that focused on the evaluation of single guidelines, the present study shows in a controlled lab experiment the combined effectiveness of 20 guidelines on real web forms. As our sample forms showed, even forms on high traffic websites can benefit from an optimization through the guidelines.

\section{Acknowledgements}

The authors would like to thank Lars Frasseck for the technical implementation, Stefan Garcia for the stimuli selection and the usability experts and all participants for their valuable contribution to this study.

Furthermore we thank: Timon Elmer, Markus Hug, Julia Kreiliger, Patrick Keller, Sébastien Orsini, Lorenz Ritzmann, Sandra Roth and Sharon Steinemann. 


\section{References}

[1] Aeberhard, A. (2011). FUS - Form Usability Scale. Development of a Usability Measuring Tool for Online Forms. Unpublished master's thesis. University of Basel, Switzerland.

[2] Brooke, J. (1996). SUS: A Quick and Dirty Usability Scale. In: P. W. Jordan, B. Thomas, B. A.

Weerdmeester \& I. L. McClelland (Eds.), Usability

Evaluation in Industry (pp. 189 - 194). London: Taylor \& Francis

[3] Bargas-Avila, J. A, Brenzikofer, O., Roth, S., Tuch, A. N., Orsini, S., \& Opwis, K. (2010). Simple but Crucial User Interfaces in the World Wide Web: Introducing 20 Guidelines for Usable Web Form Design. In: R. Matrai (Ed.), User Interfaces (pp. 1 - 10). InTech, ISBN: 978953-307-084-1.

[4] Bargas-Avila, J. A., Brenzikofer, O., Tuch, A. N., Roth, S. P., \& Opwis, K. (2011). Working towards usable forms on the World Wide Web: Optimizing date entry input fields. Advances in Human Computer Interaction, Article ID 202701.

[5] Bargas-Avila, J. A., Brenzikofer, O., Tuch, A. N. Roth, S. P., \& Opwis, K. (2011). Working towards usable forms on the World Wide Web: Optimizing multiple selection interface elements. Advances in Human Computer Interaction, Article ID 347171.

[6] Bargas-Avila, J. A., Orsini, S., Piosczyk, H., Urwyler, D., \& Opwis, K. (2010). Enhancing online forms: Use format specifications for fields with format restrictions to help respondents, Interacting with Computers, 23(1), 33 - 39

[7] Christian, L., Dillman, D., \& Smyth, J. (2007). Helping respondents get it right the first time: the influence of words, symbols, and graphics in web surveys. Public Opinion Quarterly, 71(1), 113 - 125.

[8] Couper, M., Tourangeau, R., Conrad, F., \& Crawford, S. (2004). What they see is what we get: response options for web surveys. Social Science Computer Review, 22(1), 111 - 127.
[9] Hart, S., \& Staveland, L. (1988). Development of NASA-TLX (Task Load Index): Results of empirical and theoretical research. P. A. Hancock \& N. Meshkati (Eds.). Human mental workload (pp. 139 - 183). Amsterdam: Elsevier Science.

[10] Idrus, Z., Razak, N. H. A., Talib, N. H. A., \& Tajuddin, T. (2010). Using Three Layer Model (TLM) in web form design: WeFDeC checklist development.

Computer Engineering and Applications (ICCEA), 385 389.

[11] Kaljuvee, O., Buyukkokten, O., Garcia-Molina, H., \& Paepcke, A. (2001). Efficient web form entry on PDAs. Proc. WWW, $663-672$

[12] Lewis, J. R. (1991). Psychometric evaluation of an after-scenario questionnaire for computer usability studies: The ASQ. SIGCHI Bulletin, 23(1), $78-81$.

[13] Money, A. G., Fernando, S., Elliman, T., \& Lines, L. (2010). A trial protocol for evaluating assistive online forms for older adults. Proc. ECIS, Paper 90.

[14] Pauwels, S. L., Hübscher, C., Leuthold, S., BargasAvila, J. A. \& Opwis, K. (2009). Error prevention in online forms: Use color instead of asterisks to mark required fields. Interacting with Computers, 21(4), 257 - 262.

[15] Seckler, M., Tuch, A. N., Opwis, K., \& Bargas-Avila, J. A. (2012). User-friendly Locations of Error Messages in Web Forms: Put them on the right side of the erroneous input field. Interacting with Computers, 24(3), 107 - 118.

[16] Wroblewski, L. (2008). Web Form Design: Filling in the Blanks. Rosenfeld Media. 